\title{
BACH1: A Potential Predictor of Survival in Early-Stage Lung Adenocarcinoma
}

\author{
Jin Zhou, ${ }^{1}$ Zheming Liu, ${ }^{1}$ Huibo Zhang, ${ }^{1}$ Tianyu Lei, ${ }^{1}$ Jiahui Liu, ${ }^{2}$ Yaqi Zhao, ${ }^{1}$ Yi Yao ${ }^{1}$ \\ and Qibin Song $\mathbb{D}^{1}$ \\ ${ }^{1}$ Cancer Center, Renmin Hospital of Wuhan University, Wuhan, China \\ ${ }^{2}$ Anesthesiology Department, Renmin Hospital of Wuhan University, Wuhan, China
}

Correspondence should be addressed to Yi Yao; yaoyi2018@whu.edu.cn and Qibin Song; qibinsong@whu.edu.cn

Received 15 September 2021; Accepted 25 November 2021; Published 6 January 2022

Academic Editor: Manuela Curcio

Copyright (c) 2022 Jin Zhou et al. This is an open access article distributed under the Creative Commons Attribution License, which permits unrestricted use, distribution, and reproduction in any medium, provided the original work is properly cited.

\begin{abstract}
Purpose. Recent researches showed the vital role of BACH1 in promoting the metastasis of lung cancer. We aimed to explore the value of BACH1 in predicting the overall survival (OS) of early-stage (stages I-II) lung adenocarcinoma. Patients and Methods. Lung adenocarcinoma cases were screened from the Cancer Genome Atlas (TCGA) database. Functional enrichment analysis was performed to obtain the biological mechanisms of BACH1. Gene set enrichment analysis (GSEA) was performed to identify the difference of biological pathways between high- and low-BACH1 groups. Univariate and multivariate COX regression analysis had been used to screen prognostic factors, which were used to establish the BACH1 expression-based prognostic model in the TCGA dataset. The C-index and time-dependent AUC curve were used to evaluate predictive power of the model. External validation of prognostic value was performed in two independent datasets from Gene Expression Omnibus (GEO). Decision analysis curve was finally used to evaluate clinical usefulness of the BACH1-based model beyond pathologic stage alone. Results. BACH1 was an independent prognostic factor for lung adenocarcinoma. High-expression BACH1 cases had worse OS. BACH1-based prognostic model showed an ideal C-index and $t$-AUC and validated by two GEO datasets, independently. More importantly, the BACH1-based model indicated positive clinical applicability by DCA curves. Conclusion. Our research confirmed that $\mathrm{BACH} 1$ was an important predictor of prognosis in early-stage lung adenocarcinoma. The higher the expression of BACH1, the worse OS of the patients.
\end{abstract}

\section{Introduction}

Lung cancer (LC) is one of the malignant tumors that threatens the health and life of human being [1]. In the past 50 years, many countries have reported a significant increase in lung cancer morbidity and mortality [2], which accounts for the first place in all malignant tumors in male and the second place in female $[2,3]$. Among all the pathological phenotypes of LC, non-small-cell lung cancer (NSCLC) presents with the highest morbidity, especially lung adenocarcinoma [4].

Transcription factors are proteins that bind to DNA regulatory sequences to modulate gene transcription, which may result in alteration in gene transcription, protein synthesis, and cellular function. Transcriptional activators promote gene transcription and repressors inhibit that of Reference
[5]. BTB and CNC homology 1 (BACH1) belongs to the bZIP [6] transcription factor family [7]. BACH1 mRNA is highly expressed in subsets of monocytes, macrophages, neutrophils, and dendritic cells, which are abundant in the tumor microenvironment (TME) [5]. As these immune cells orchestrate nearly all of the proliferation, differentiation, and metastasis in the development of solid tumor, the TME system has been recognized as the most promising antitumor therapy [8].

As it is reported recently, the reactive oxygen system (ROS) has been defined as another important factor in the tumor tissue homeostasis and cellular differentiation and metastasis. Antioxidant transcription factor NRF2 are abundant in cancer, which suggested that increased antioxidant defense contributes to the tumor progression. ROS plays a vital role in the homeostasis in normal or tumor tissue and 
TABLe 1: Demographic and clinical characteristics of early-stage lung adenocarcinoma cases.

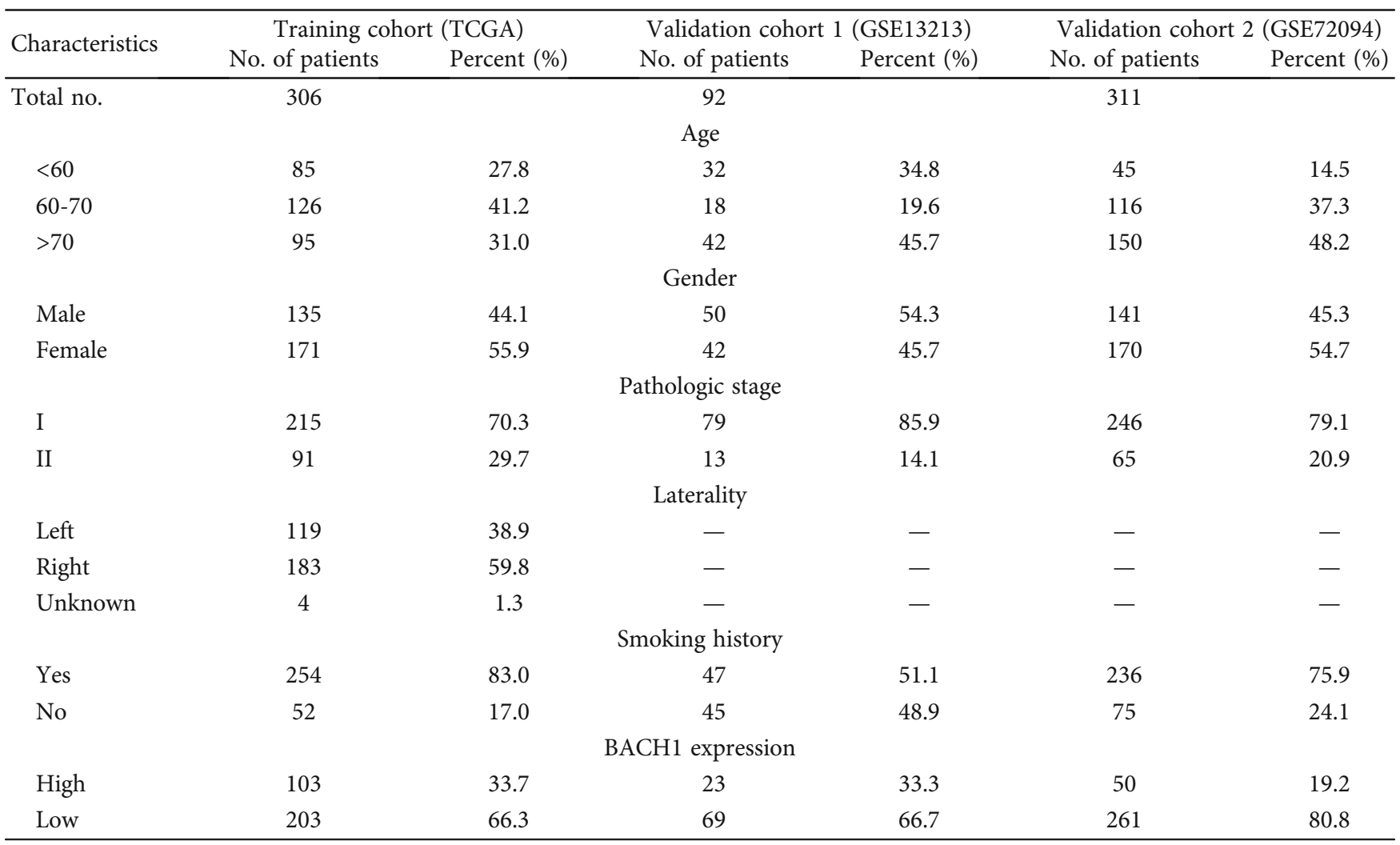

hints its contradictory and complex role in the TME system [9]. Lee et al. found that heme can inhibit the development of triple-negative breast cancer (TNBC) through the suppression of activation of BACH1, which can negatively modulate the gene expression of electron transport chain (ETC) in mitochondria [10]. Lignitto et al. [11] and Wiel et al. [12] groups reported NRF2 activation in KrasG12D; p53flox/flox lung tumor mouse model can indirectly promote the stability of BACH1 via the inhibition of heme and at last induce the metastasis of lung cancer, which may be contributed by antioxidant treatment.

The above studies indicated that the upregulation of $\mathrm{BACH} 1$ promoted lung cancer metastasis. However, there is no direct evidence of $\mathrm{BACH} 1$ expression in relation to the prognosis of early-stage lung cancer.

We utilized bioinformatics approach such as the Cancer Genome Atlas (TCGA) and Gene Expression Omnibus (GEO) database to explore the role of $\mathrm{BACH} 1$ expression in the prognosis of early-stage lung adenocarcinoma. In this study, we established a BACH1-related prognostic model to predict overall survival (OS) of early-stage lung adenocarcinoma. In addition, bioinformatics analyses were performed to explore the biological processes and possible cell signal pathways underlying the prognosis.

\section{Materials and Methods}

2.1. Data Acquisition. The gene expression data and corresponding clinical information of lung adenocarcinoma were screened from the Cancer Genome Atlas (TCGA) website (https://portal.gdc.cancer.gov/repository) (up to May 17, 2020). 515 cases with RNA-sequencing data and clinical information were initially downloaded. The gene expression profiles were normalized by variance stabilizing transformation (VST) using DESeq2 R package. 134 cases with less than 30 days of following-up time and 75 cases with unknown clinical stage or stages III-IV were excluded; eventually, 306 cases were enrolled for subsequent analysis.

2.2. Functional Enrichment Analysis. Spearman correlation analysis was performed between the expression of $\mathrm{BACH} 1$ and other encoding genes, and then genes with $P$ value less than 0.05 and the highest correlation coefficient $(>0.3)$ were selected. Gene Ontology (GO) analysis was performed using the clusterProfiler R package [13] to evaluate the BACH1related biological process (BP), cellular compartment (CC), and molecular function (MF). Kyoto Encyclopedia of Genes and Genomes (KEGG) analysis was conducted to further evaluate potential biological signal pathways related to BACH1 expression. We visualized significant processes and pathways using the function of clusterProfiler R package.

2.3. Gene Set Enrichment Analysis. Gene set enrichment analysis (GSEA) was performed to identify the difference of biological pathways and corresponding genes between lung adenocarcinoma cases with the high- and low-BACH1 groups, in order to further evaluate the potential mechanism of the underlying involvement of BACH1 in lung 


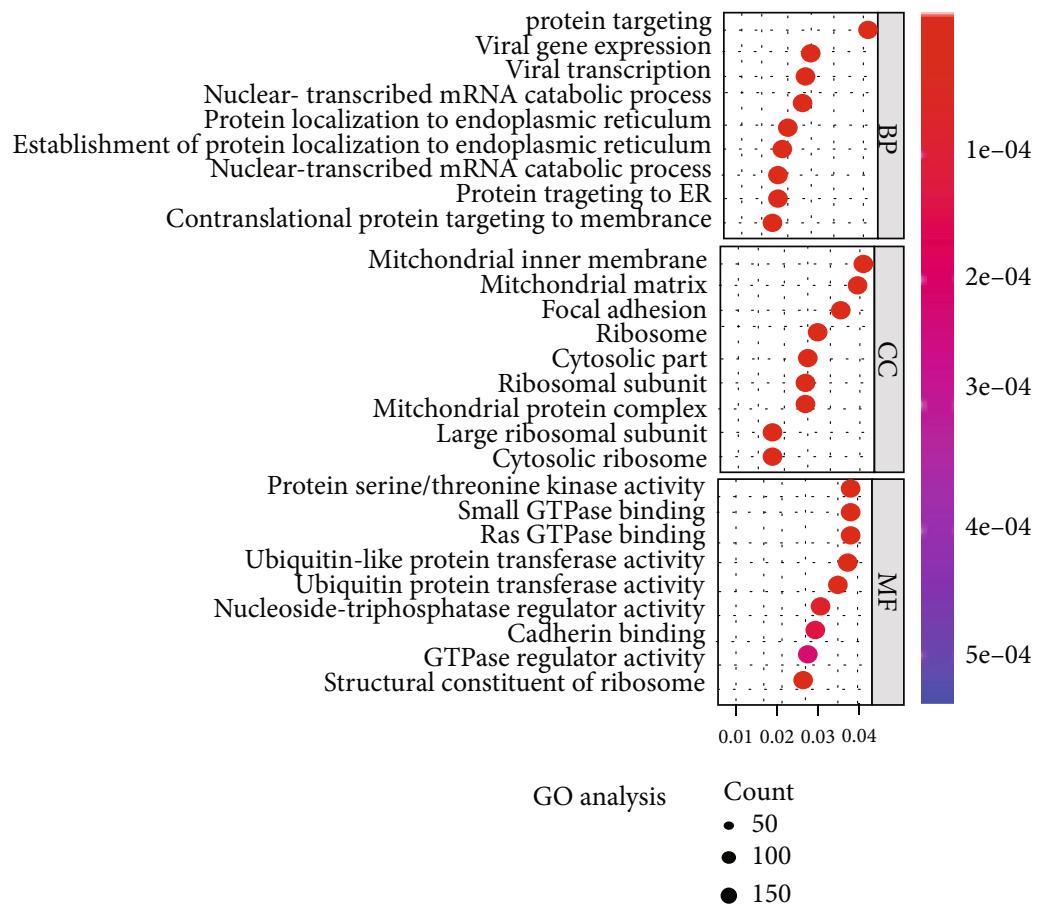

(a)

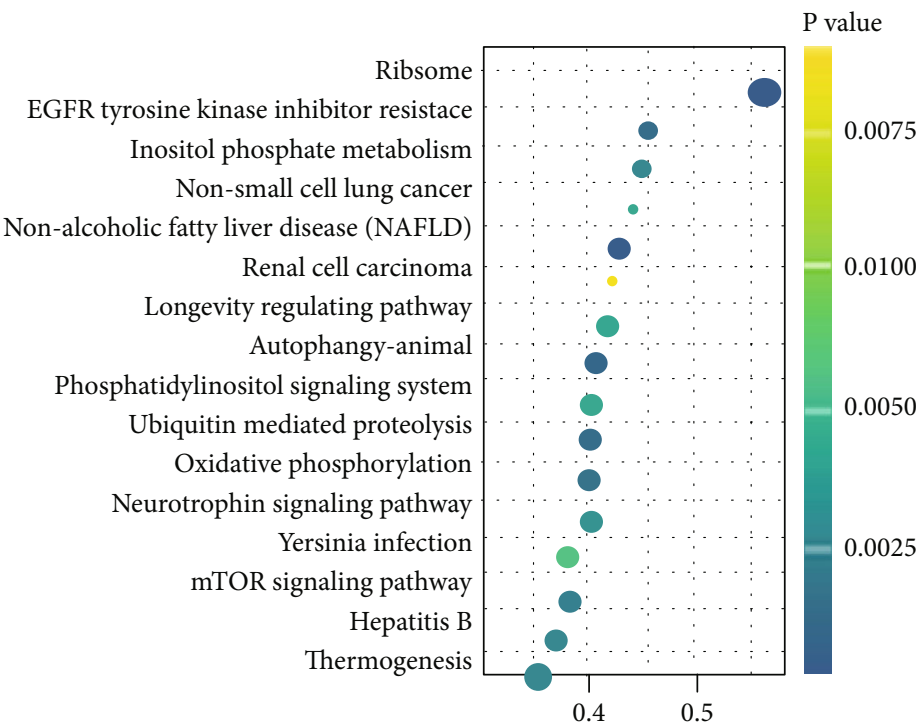

Rich factor

KEGG analysis
Count
- 50
- 150
- $100 \quad 125$

(b)

FIgUre 1: Continued. 


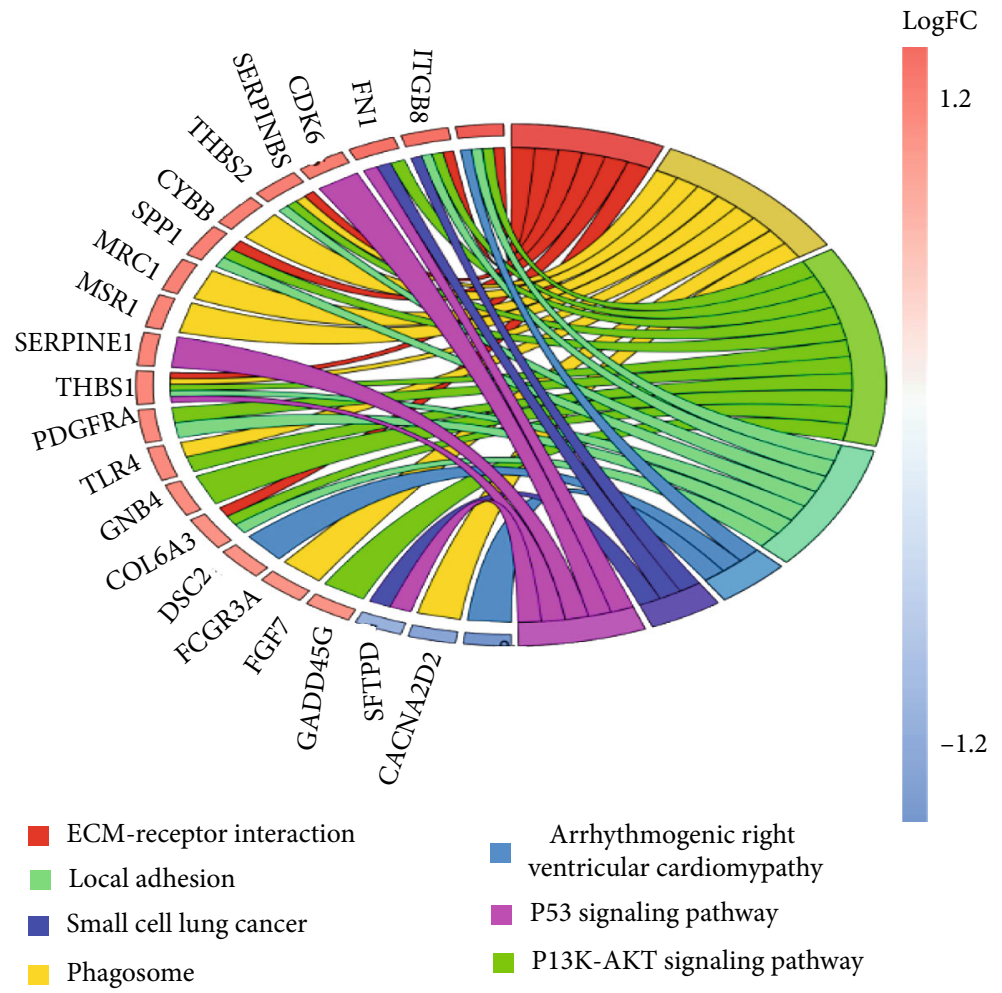

(c)

FIGURE 1: GO and KEGG analysis of BACH1 in the training set (TCGA dataset). (a) GO analysis of the BACH1-related BP, CC, and MF. (b) KEGG analysis of potential biological signal pathways related to BACH1 expression. (c) Circular plot of the KEGG pathways enriched for $\mathrm{BACH} 1$ expression-related genes.

adenocarcinoma prognosis. An annotated gene set file (h.all.v7.1.entrez.gmt) was selected as reference. The threshold was set at $P<0.05$.

\subsection{Construction of BACH1 Expression-Based Prognostic} Model. BACH1 expression data and clinical information were integrated to analyze the relationship between $\mathrm{BACH} 1$ and OS. Univariate and multivariate COX regression analyses were conducted to select prognostic factors. Then, variables that achieved significance at $P<0.05$ after the multivariable analysis were screened to establish the nomogram model. Concordance index (C-index) was used to quantify the predictive accuracy of the model. C-index ranges from 0.5 , which means a random chance, to 1.0 , which indicates a perfect ability of correct prediction. The calibration plot was performed to compare actual and predicted probability of 3- and 5-year OS. Then, the reliability of the model was verified by means of the time-dependent area under ROC curves ( $t$-AUC). A $t$-AUC value above 0.7 suggests that a reasonable prediction model has been constructed.

2.5. External Validation of Prognostic Model. We systematically searched for gene expression datasets of early-stage lung adenocarcinoma that were published and available in Gene Expression Omnibus (GEO) website (https://ncbi .nlm.nih.gov/geo). We finally selected two cohorts of samples in GEO databases (GSE13213 and GSE72094) as exter- nal validation cohorts to further validate the value of BACH1 expression-based prognostic model.

2.6. Risk Group Stratification Based on the Nomogram and Clinical Usefulness. Log-rank statistics was used to make a risk group stratification according to the total risk scores based on the nomogram, in order to illustrate the independent discrimination ability of BACH1-based model beyond BACH1 alone. Decision curve analysis (DCA) [14, 15] was finally used to evaluate clinical usefulness of BACH1-based model beyond pathologic stage alone.

All analyses were conducted in $\mathrm{R}$ software (version 3.6.1). The value of $P<0.05$ was statistically significant.

\section{Results and Discussion}

3.1. Characteristics of Cases in TCGA Dataset. A total of 276 cases of TCGA cohort with both clinical and gene expression data were enrolled in the present study (Table 1). The median follow-up time was 20 months, and median age was 66 years old. $44.1 \%$ cases were male. The pathologic stage included 215 (70.3\%) with stage I and 91 (29.7\%) with stage II. Most cases (254, 83.0\%) had smoking history. 48 of $204(23.5 \%)$ cases had lymph node metastases (pelvic and para-aortic). Cases with high- and low-expression BACH1 accounted for $33.7 \%$ (103) and 66.3\% (203), respectively. 
TABLE 2: Gene sets enriched in high-expression BACH1 cases.

\begin{tabular}{|c|c|c|c|}
\hline Gene set name/signal pathway & NES & $P$ value & $Q$ value \\
\hline HALLMARK_OXIDATIVE_PHOSPHORYLATION & -2.575 & 0.002 & 0.002 \\
\hline HALLMARK_MYC_TARGETS_V1 & -1.448 & 0.002 & 0.002 \\
\hline HALLMARK_DNA_REPAIR & -1.981 & 0.002 & 0.002 \\
\hline HALLMARK_MYC_TARGETS_V2 & -1.964 & 0.002 & 0.002 \\
\hline HALLMARK_ANGIOGENESIS & 2.114 & 0.002 & 0.002 \\
\hline HALLMARK_ANDROGEN_RESPONSE & 1.783 & 0.002 & 0.002 \\
\hline HALLMARK_PROTEIN_SECRETION & 1.657 & 0.002 & 0.002 \\
\hline HALLMARK_IL6_JAK_STAT3_SIGNALING & 1.908 & 0.002 & 0.002 \\
\hline HALLMARK_TGF_BETA_SIGNALING & 1.860 & 0.002 & 0.002 \\
\hline HALLMARK_HYPOXIA & 1.559 & 0.003 & 0.002 \\
\hline HALLMARK_G2M_CHECKPOINT & 2.300 & 0.003 & 0.002 \\
\hline HALLMARK_COMPLEMENT & 1.915 & 0.003 & 0.002 \\
\hline HALLMARK_APOPTOSIS & 1.411 & 0.003 & 0.002 \\
\hline HALLMARK_E2F_TARGETS & 1.589 & 0.003 & 0.002 \\
\hline HALLMARK_INTERFERON_GAMMA_RESPONSE & 1.884 & 0.003 & 0.002 \\
\hline HALLMARK_MITOTIC_SPINDLE & 2.151 & 0.003 & 0.002 \\
\hline HALLMARK_TNFA_SIGNALING_VIA_NFKB & 2.265 & 0.003 & 0.002 \\
\hline HALLMARK_APICAL_JUNCTION & 1.645 & 0.003 & 0.002 \\
\hline HALLMARK_IL2_STAT5_SIGNALING & 1.877 & 0.003 & 0.002 \\
\hline HALLMARK_KRAS_SIGNALING_UP & 2.349 & 0.003 & 0.002 \\
\hline HALLMARK_MTORC1_SIGNALING & 1.372 & 0.003 & 0.002 \\
\hline HALLMARK_EPITHELIAL_MESENCHYMAL_TRANSITION & 3.165 & 0.003 & 0.002 \\
\hline HALLMARK_INFLAMMATORY_RESPONSE & 2.423 & 0.003 & 0.002 \\
\hline HALLMARK_PEROXISOME & -1.526 & 0.010 & 0.006 \\
\hline HALLMARK_XENOBIOTIC_METABOLISM & -1.393 & 0.015 & 0.009 \\
\hline HALLMARK_COAGULATION & 1.291 & 0.024 & 0.013 \\
\hline HALLMARK_HEDGEHOG_SIGNALING & 1.536 & 0.026 & 0.014 \\
\hline HALLMARK_REACTIVE_OXYGEN_SPECIES_PATHWAY & -1.481 & 0.032 & 0.016 \\
\hline
\end{tabular}

NES: normalized enrichment score; NOM: nominal. Gene sets with $P$ value $<0.05$ and $Q$ value $<0.05$ are considered as significant.

3.2. Functional Enrichment Analysis. GO and KEGG analysis was performed to obtain a novel understanding of biological mechanisms of BACH1. 5000 genes that highly associated with BACH1 (correlation coefficient $>0.3$ and $P<0.05$ ) were extracted and subjected to GO and KEGG analyses. Genes related to BACH1 expression were mainly enriched in BP column of "protein targeting", "nuclear-transformed mRNA catabolic process", "protein localization to endoplasmic reticulum", "protein targeting to endoplasmic reticulum", "cotranslational protein targeting to membrane" terms, and in CC column of "mitochondrial inner membrane", "mitochondrial matrix", "focal adhesion", "ribosome", "cytosolic part" terms, and in MF column of "protein serine/threonine kinase activity", "small GTPase binding”, "RAS GTPase binding”, "ubiquitin-protein transferase activity", "nucleoside-triphosphatase regulator activity", "cadherin binding", "GTPase regulator activity", and "structural constituent of ribosome" terms according to the GO analysis (Figure 1(a)), as well as "ribosome", "EGFR tyrosine kinase inhibitor resistance", "inositol phosphate metabolism", "non-small-cell lung cancer", "autophagy", "oxidative phosphorylation", and "mTOR signaling pathway" according to KEGG analysis (Figure 1(b)). Furtherly, we screened out 200 genes with the most significant correlation with $\mathrm{BACH} 1$ expression to construct circular plot of KEGG and found that the PI3K-Akt signaling pathway, which was known to be a signaling pathway closely related to the occurrence and development of tumors, was significantly activated (Figure 1(c)) in the high-expression group of BACH1.

3.3. Potential Mechanism Underlying the Role of BACH1 Affecting Prognosis. GSEA was performed to identify the difference of biological pathways and corresponding genes between 103 high- and 203 low-expression BACH1 cases. 28 biological processes were significantly enriched (Table 2), 21 activated, and 7 suppressed cell signal pathways.

We selected the most significantly enriched pathways based on normalized enrichment score (NES) in BACH1 high-expression phenotype. The results revealed that OXIDATIVE_PHOSPHORYLATION $(\mathrm{NES}=-2.575, \quad P=0.002)$, MYC_TARGETS_V1 $(\mathrm{NES}=-1.448, \quad P=0.002), \quad$ DNA_ 


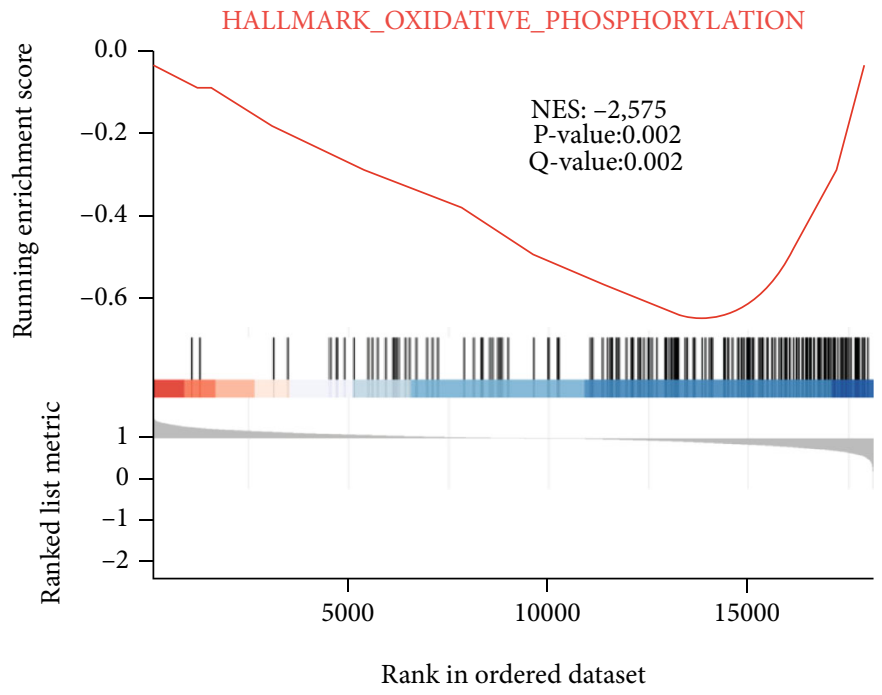

(a)

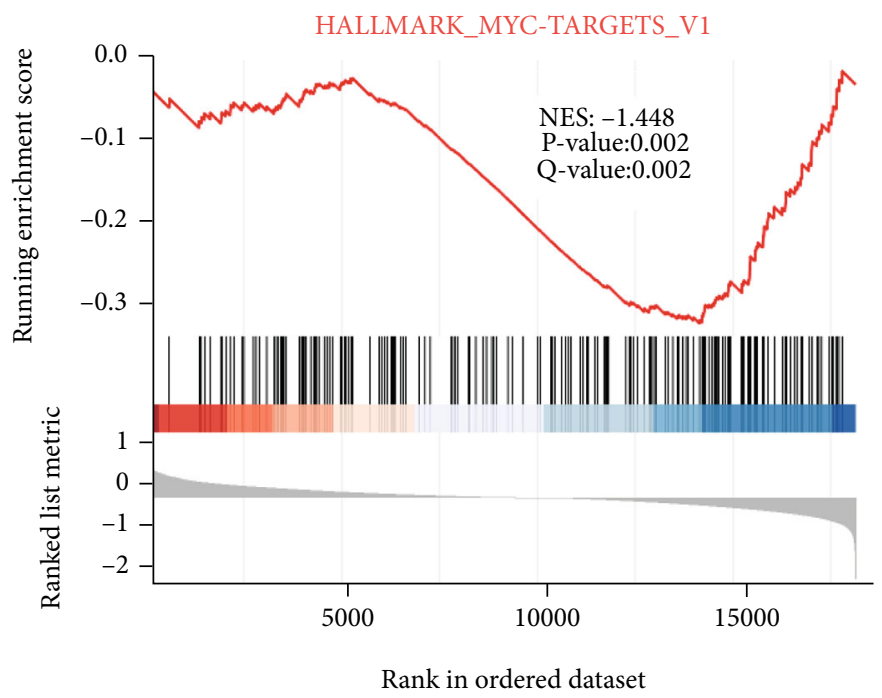

(b)

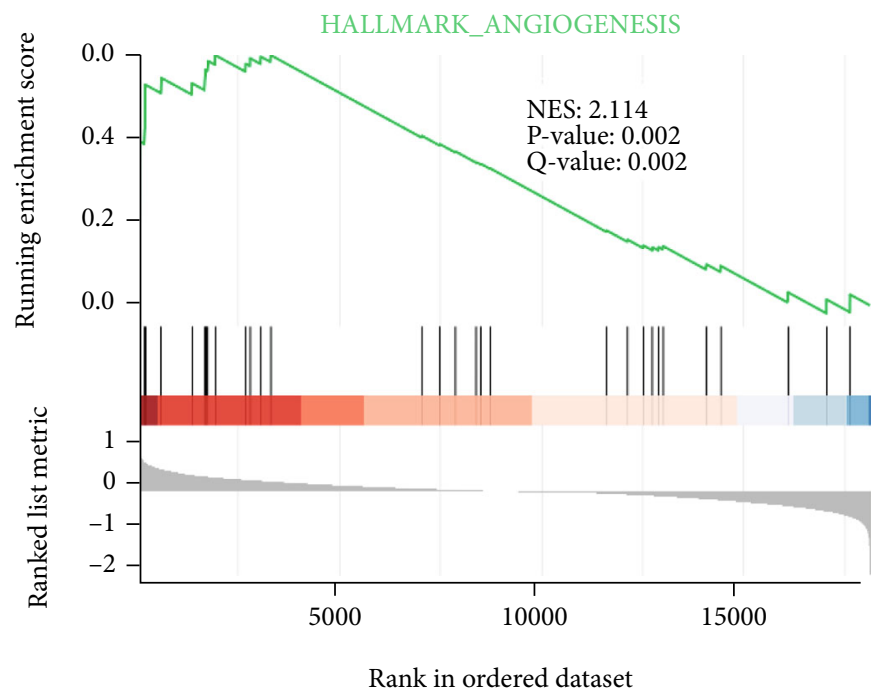

(c)

Figure 2: Continued. 


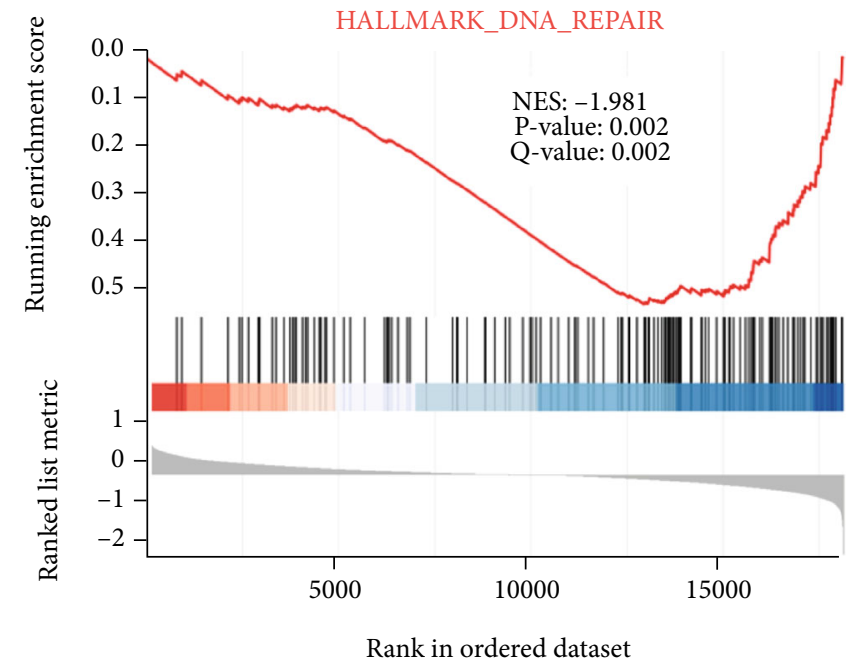

(d)

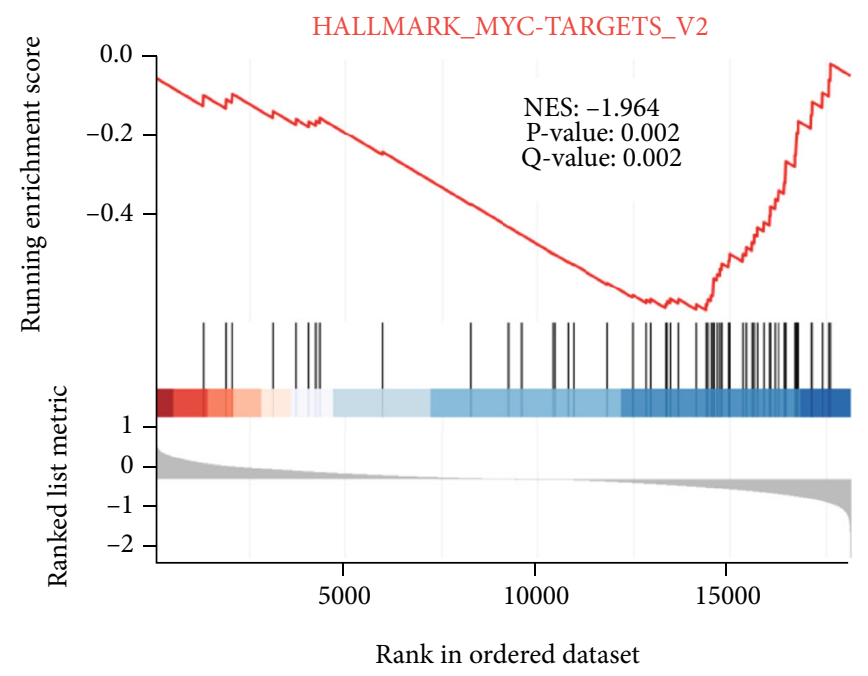

(e)

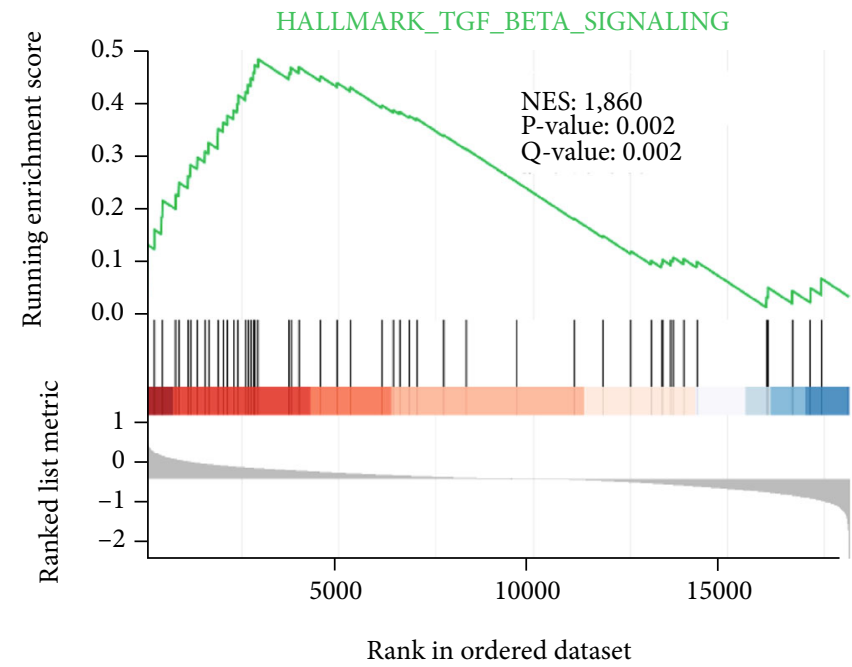

(f)

FIGURE 2: GSEA analysis of BACH1 in the training set (TCGA dataset). Significant enrichment of the BACH1-related signaling pathways in the high-expression BACH1 group compared with that in the low-expression BACH1 group. 
TABLE 3: Univariate and multivariate Cox analyses of OS in training cohort.

\begin{tabular}{|c|c|c|c|c|c|c|}
\hline \multirow{2}{*}{ Characteristics } & \multicolumn{3}{|c|}{ Univariable analysis } & \multicolumn{3}{|c|}{ Multivariable analysis } \\
\hline & HR & 95\% CI & $P$ & HR & $95 \%$ CI & $P$ \\
\hline Age & & & 0.002 & & & 0.001 \\
\hline$<60$ & 1.000 & & & 1.000 & & \\
\hline $60-70$ & 0.698 & $0.313-1.556$ & 0.379 & 0.674 & $0.299-1.521$ & 0.342 \\
\hline$>70$ & 2.290 & $1.143-4.587$ & 0.019 & 2.355 & $1.145-4.841$ & 0.020 \\
\hline Gender & & & 0.292 & & & \\
\hline Male & 1.000 & & & & & \\
\hline Female & 1.227 & $0.695-2.167$ & 0.480 & & & \\
\hline Pathologic stage & & & $<0.001$ & & & $<0.001$ \\
\hline I & 1.000 & & & 1.000 & & \\
\hline II & 2.673 & $1.516-4.712$ & 0.001 & 2.819 & $1.578-5.036$ & $<0.001$ \\
\hline Laterality & & & 0.582 & & & \\
\hline Right & 1.000 & & & & & \\
\hline Left & 1.118 & $0.632-1.975$ & 0.475 & & & \\
\hline Unknown & 0.991 & $0.134-7.343$ & 0.993 & & & \\
\hline Smoking history & & & 0.375 & & & \\
\hline Yes & 1.000 & & & & & \\
\hline No & 1.195 & $0.610-2.341$ & 0.603 & & & \\
\hline BACH1 expression & & & $<0.001$ & & & $<0.001$ \\
\hline High & 1.000 & & & 1.000 & & \\
\hline Low & 0.284 & $0.158-0.511$ & $<0.001$ & 0.315 & $0.174-0.571$ & $<0.001$ \\
\hline
\end{tabular}

REPAIR (NES $=-1.981, P=0.002)$, and MYC_TARGETS_ V2 (NES $=-1.964, P=0.002)$ pathways were differentially suppressed. ANGIOGENESIS (NES $=2.114, P=0.002$ ), IL6_ JAK_STAT3_SIGNALING $(\mathrm{NES}=1.908, P=0.002)$, and TGF_BETA_SIGNALING $(\mathrm{NES}=1.860, P=0.002)$ pathways were differentially activated (Figure 2).

\section{Development and Validation of BACH1- Based Prognostic Model}

Univariate and multivariate COX regression analyses were used to select risk factors. According to Cox regression analysis (Table 3$)$, age $(P<0.001)$, pathologic stage $(P<0.001)$, and BACH1 expression $(P<0.001)$ were significantly independent prognostic factors and were incorporated to establish the nomogram model (Figure 3(a)).

The predictive ability of the model was then evaluated in TCGA dataset and independently validated in the validation cohort of GSE13213 and GSE72094.

The C-index of the model was 0.782 (95\% CI [0.752, 0.812]) in TCGA dataset while 0.648 (95\% CI [0.596, $0.700]$ ) in GSE13213 cohort and 0.632 (95\% CI [0.595, 0.669]) in GSE72094 cohort. Figure 3(b) shows that $t$-AUC value was above 0.6 for the prediction of deterioration risk within 5 years both in TCGA dataset and validation cohort, indicating that a stable prognostic model was established.

Furthermore, the calibration curves of the model showed high consistencies between predicted and observed 3- and 5year OS probability in TCGA dataset and GSE13213 cohort
(Figures 3(c), 3(d), 3(f), and 3(g)) and 2- and 3-year OS probability in GSE72094 cohort. Thus, the model showed considerably discriminative and calibrating abilities.

\section{Risk Stratification Based on the Nomogram}

High-expression BACH1 cases had worse OS according to Kaplan-Meier curves (TCGA dataset, $P<0.001$; GSE13213 cohort, $P=0.012$; GSE72094 cohort, $P=0.003$ ) (Figures 4(a), 4(d), and 4(g)). Risk stratification was made based on the nomogram. The total point of each case was counted based on the score of each variable. Cases were grouped into two risk groups according to total points by utilizing X-tile software: low-risk (total points $<10$ ) and high-risk (total points $\geq 10$ ) group. The risk plot showed that the deaths occurred more frequently in the high-risk group in both TCGA and validation cohort (Figures 4(b), 4(e), and 4(h)). The Kaplan-Meier curves also presented the significant discrimination among two risk groups both in TCGA $(P<0.001)$ and validation cohort (GSE132123, $P=$ 0.003; GSE72094, $P=0.002)$.

5.1. Clinical Usefulness. DCA was applied to evaluate the clinical usefulness of the model by quantifying the net benefit at different threshold probabilities compared with stage systems (Figure 5). The model showed more net benefits than stage systems across a wider range of threshold probabilities both in TCGA dataset and validation cohort. 
Points

Age

Stage

BACH1

Total points

3-Year os probability

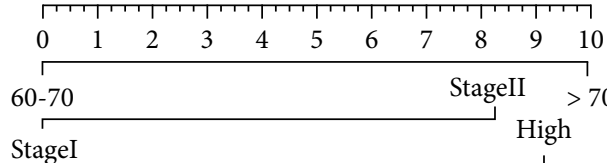

StageI

Low

5-Year os probability

\begin{tabular}{lllllllll}
\hline & 5 & 1 & 1 & 1 & 1 & \\
0 & 5 & 10 & 15 & 20 & 25 & 30
\end{tabular}

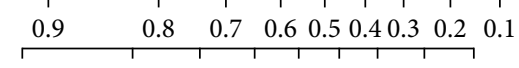

0.9

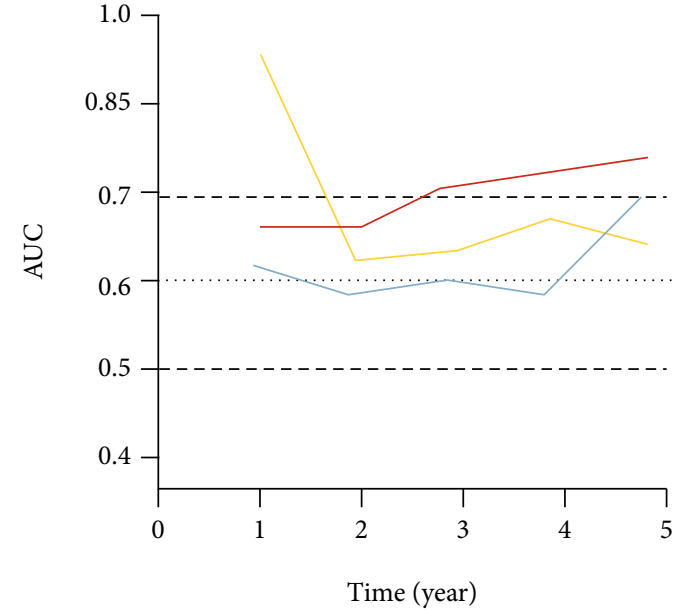

— Training cohort

Validation cohort1 (GSE13213)

Validation cohort2 (GSE72094)

(b)

(a)

3-year OS (training cohort)

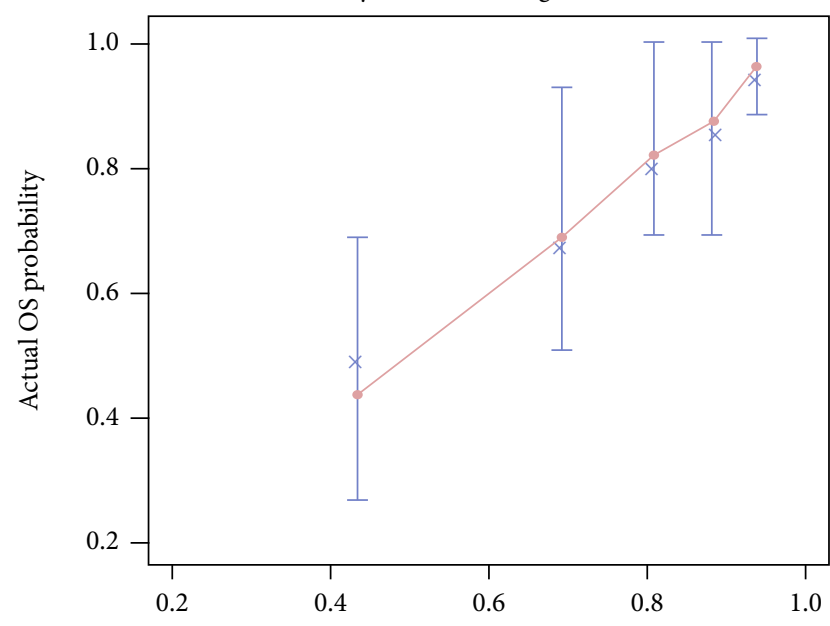

Nomogram-predicted OS probability

(c) 3-year os (validation cohort1)

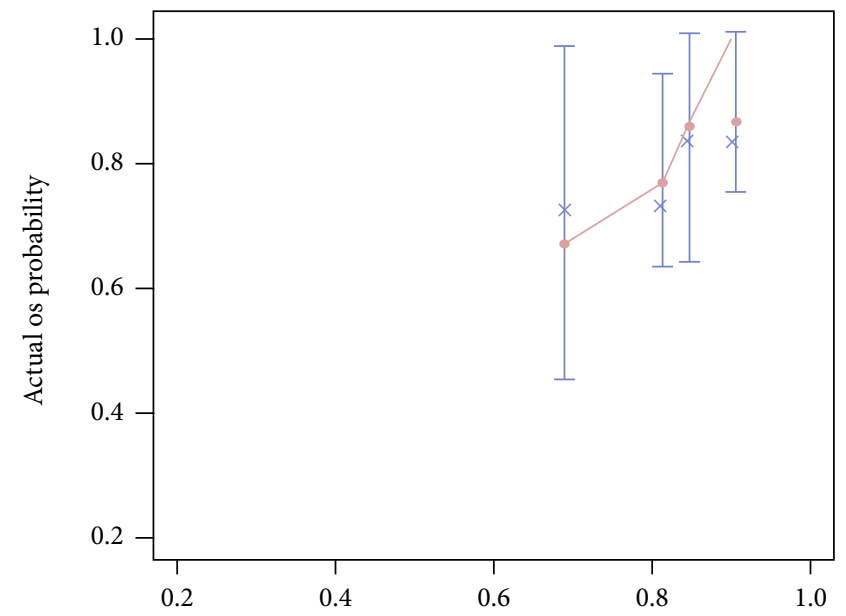

Nomogram-predicted os probability

(d)

Figure 3: Continued. 


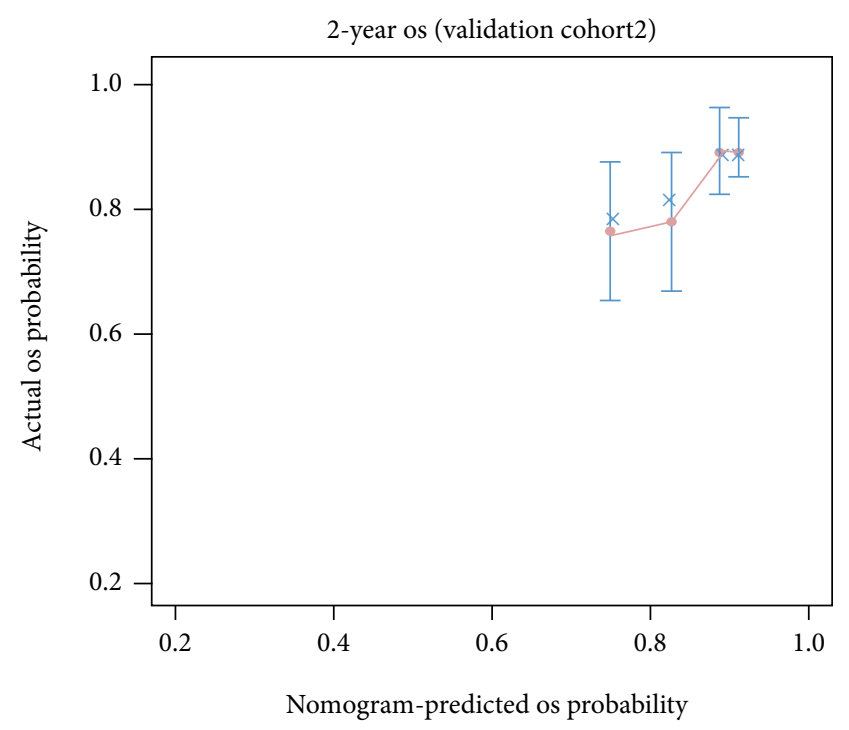

(e)

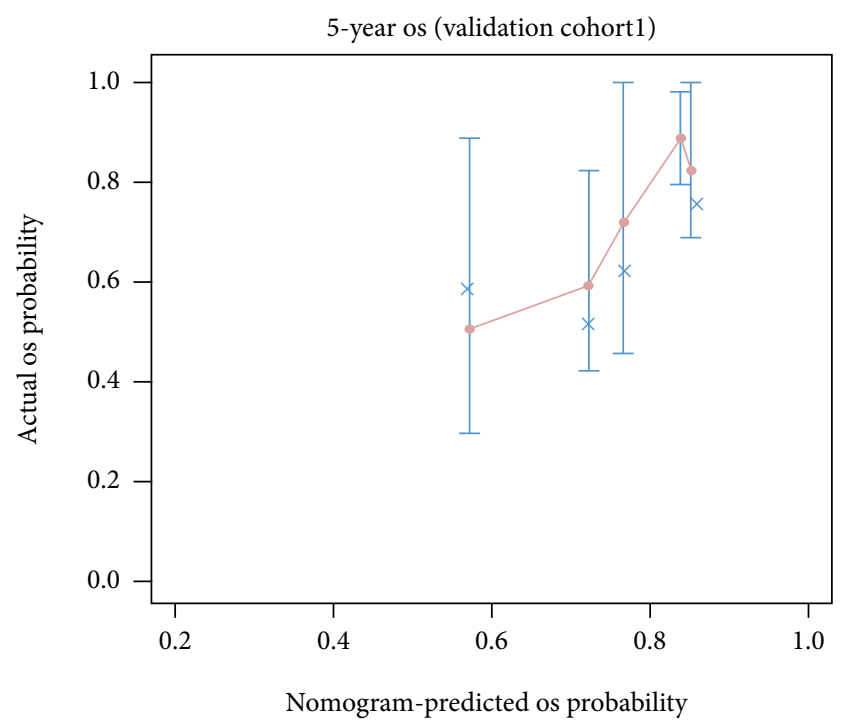

(g)

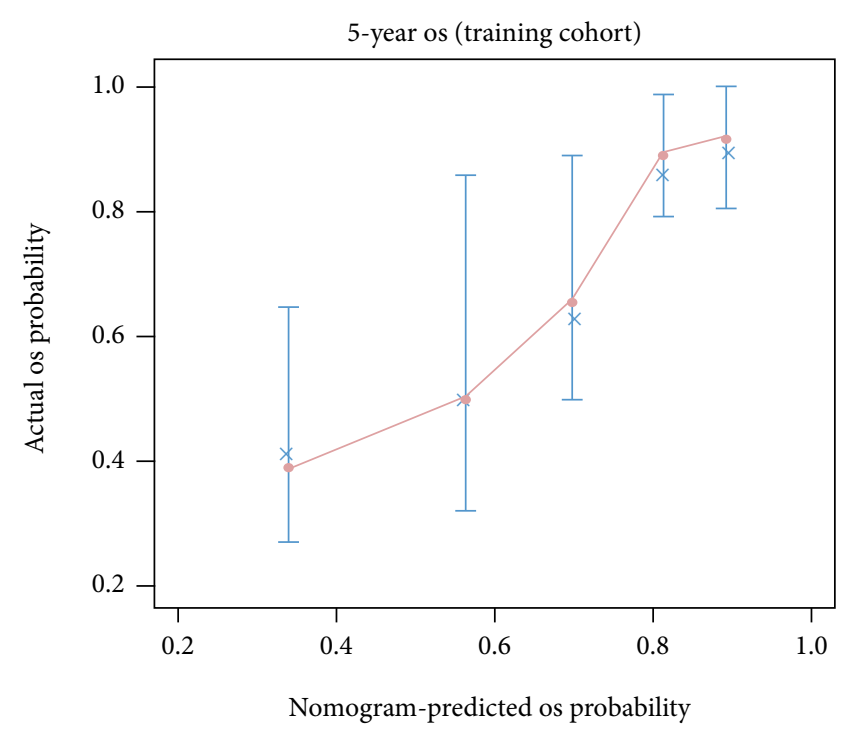

(f)

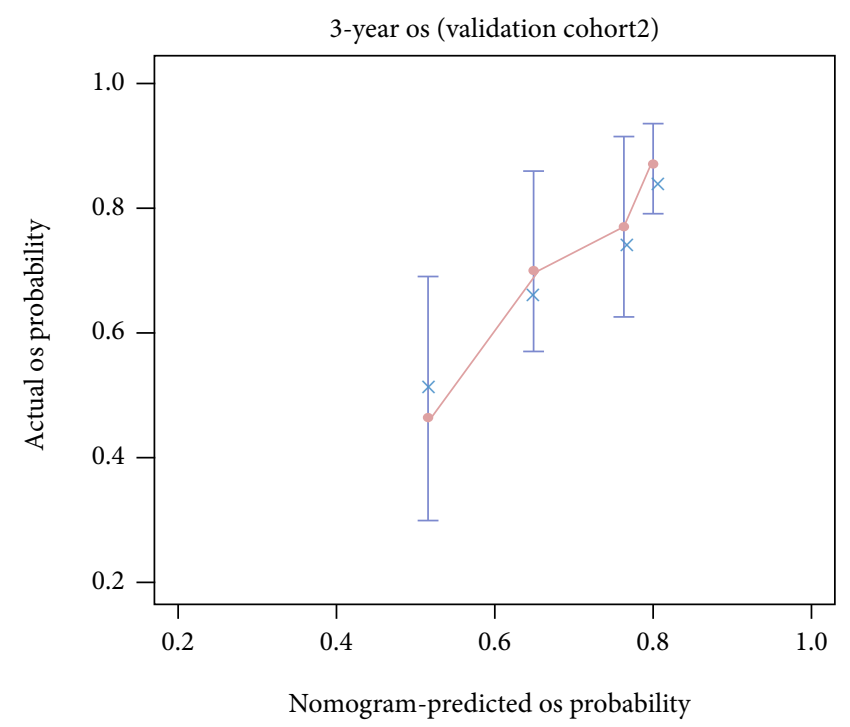

(h)

FIgURE 3: Nomogram construction and evaluation. (a) A constructed nomogram for risk prediction of OS. (b) T-AUC of the nomogram model in the TCGA dataset and validation cohort (GSE13213, GSE72094). (c) Calibration curves of the 3-year OS in the TCGA dataset. (d) Calibration curves of the 3-year OS in the validation cohort 1 (GSE13213). (e) Calibration curves of the 2-year OS in the validation cohort 2 (GSE72094). (f) Calibration curves of the 5-year OS in the TCGA dataset. (g) Calibration curves of the 5-year OS in the validation cohort 1 (GSE13213). (h) Calibration curves of the 3-year OS in the validation cohort 2 (GSE72094).

\section{Discussion}

In this pioneering study, we confirmed that BACH1 was an important prognostic factor for early-stage lung adenocarcinoma by establishing a BACH1-based prognostic model that incorporated BACH1 expression and clinical characteristics. The prognostic model was evaluated by a variety of statistical indicators and validated by independent datasets and proved to be accurate. More importantly, the BACH1based model indicated positive clinical applicability by DCA curves.

Countless molecular factors contribute to the proliferation and metastasis of cancer, which meant its thousand years living as one of the most malignant diseases with us human beings. BACH1 on behalf of bad prognosis gene has been elucidated by the presentation of its biofunction and molecular mechanism. Lignitto et al. [11] and Wiel et al. [12] groups reported that NRF2 activation can indirectly promote the stability of $\mathrm{BACH} 1$ and at last induce the metastasis of lung cancer; however, no research had ever illustrated whether BACH1 affects the prognosis of earlystage lung cancer. We firstly confirmed the vital role of $\mathrm{BACH} 1$ in the prognosis of early-stage lung cancer.

With the highest mortality, lung adenocarcinoma also shows more gene mutation and leads to its wide variety of treatments [16, 17]. BACH1-associated gene enrichment 

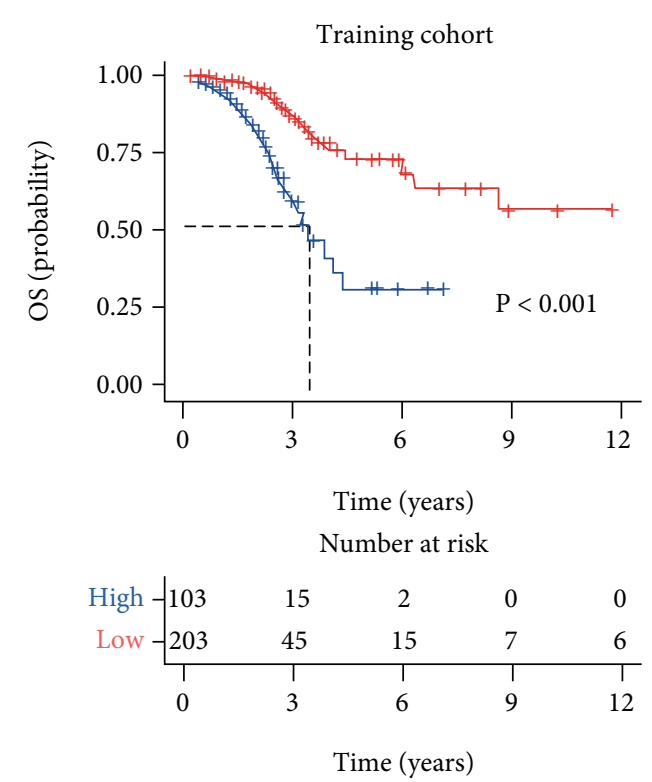

BACH1 expression

$$
\begin{aligned}
& + \text { High } \\
& + \text { Low }
\end{aligned}
$$

(a)

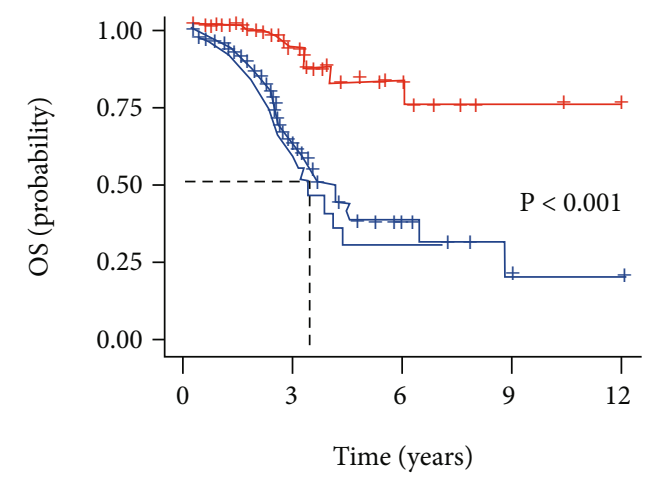

Number at risk

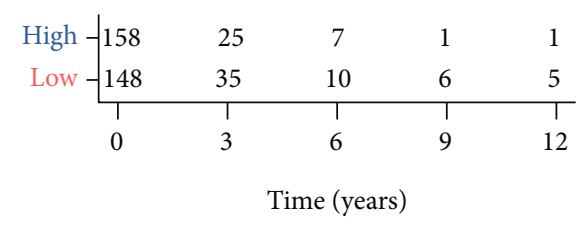

Nomogram risk stratification

$$
\begin{aligned}
& + \text { High } \\
& + \text { Low }
\end{aligned}
$$

(c)

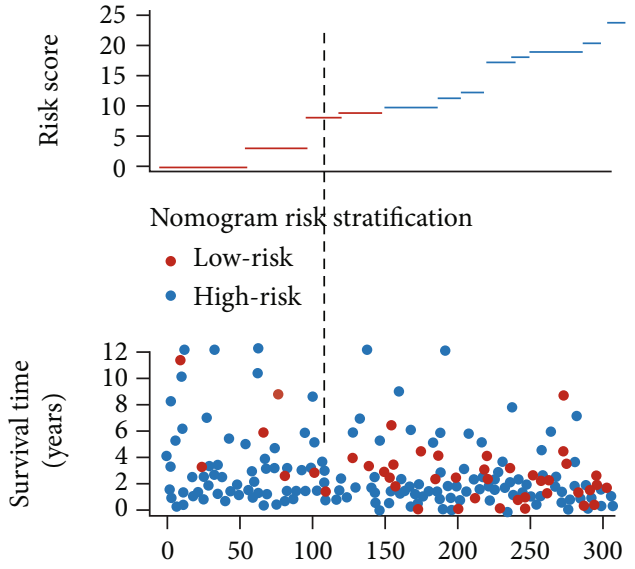

Patients (increasing nomogram score)

- Dead Nomogram risk stratification

- Alive High-risk

Low-risk

(b)

Validation cohort 1 (GSE13213)

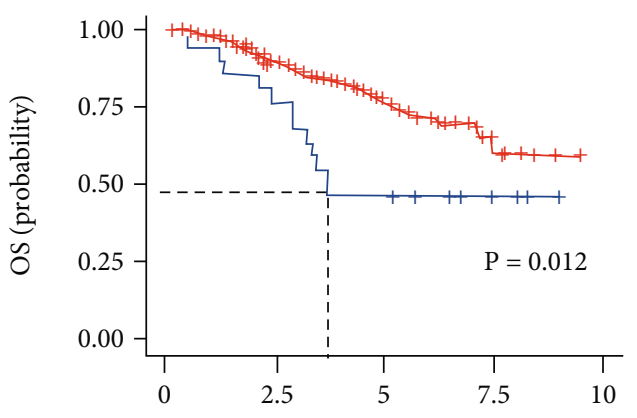

Time (years)

Number at risk

How -\begin{tabular}{ccccc}
23 & 18 & 11 & 4 & 0 \\
69 & 64 & 54 & 14 & 0 \\
\hline 1 & 1 & 1 & 1 & 1 \\
0 & 2.5 & 5 & 7.5 & 10 \\
\multicolumn{5}{c}{ Time (years) }
\end{tabular}

BACH1 expression

+ High

(d)

Figure 4: Continued. 


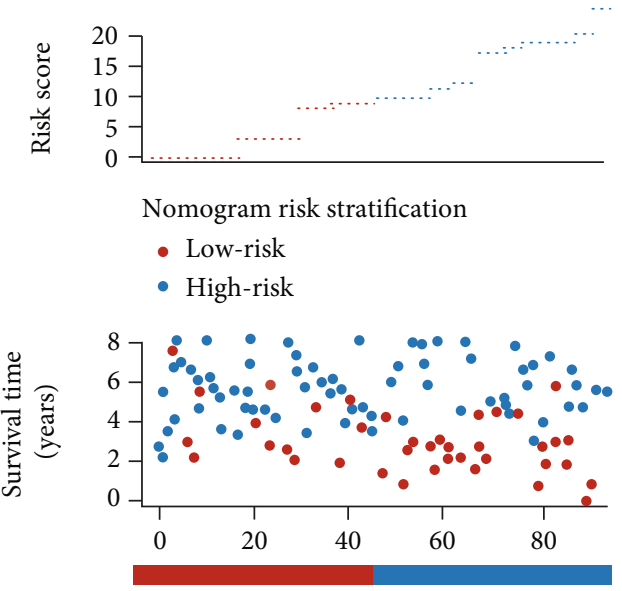

Patients (increasing nomogram score)

$\begin{array}{lc}\text { - Dead } & \text { Nomogram risk stratification } \\ \text { - Alive } & \text { High-risk } \\ & \text { Low-risk }\end{array}$

Validation cohort 2 (GSE72094)

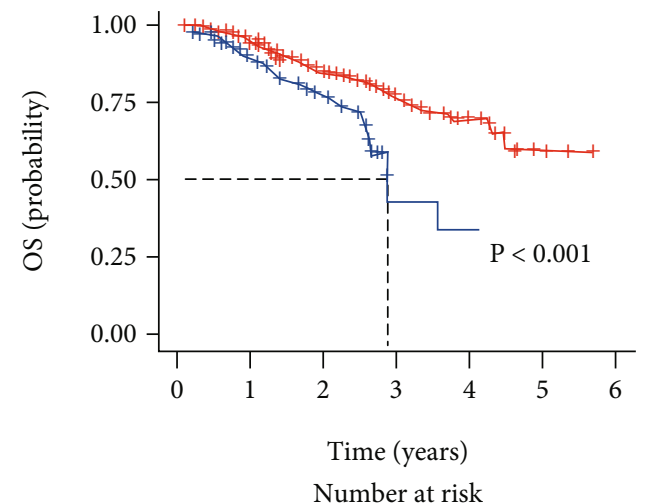

Low -\begin{tabular}{ccccccc}
50 & 41 & 30 & 5 & 1 & 0 & 0 \\
261 & 232 & 177 & 56 & 26 & 4 & 0 \\
\hline 1 & 1 & 1 & 3 & 4 & 5 & 6 \\
0 & 1 & 2 & 3 & \multicolumn{5}{c}{ Time (years) }
\end{tabular}

BACH1 expression

$$
+ \text { High }
$$

(g)

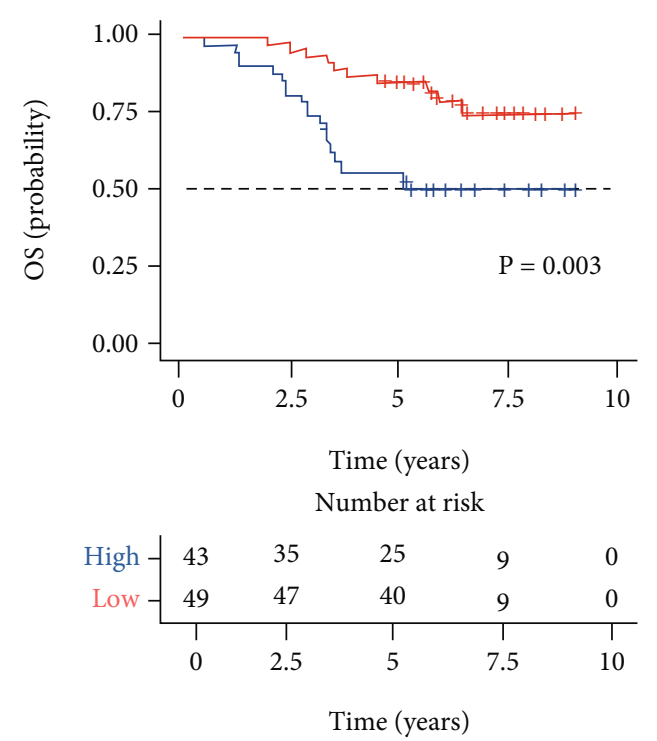

Nomogram risk stratification

$$
\begin{aligned}
& + \text { High } \\
& + \text { Low }
\end{aligned}
$$

(f)

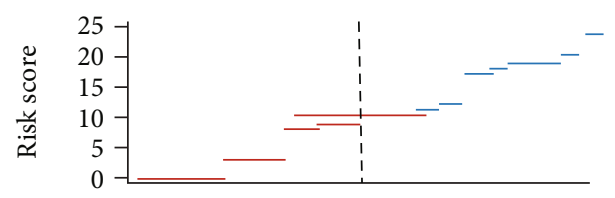

Nomogram risk stratification

- Low-risk

- High-risk

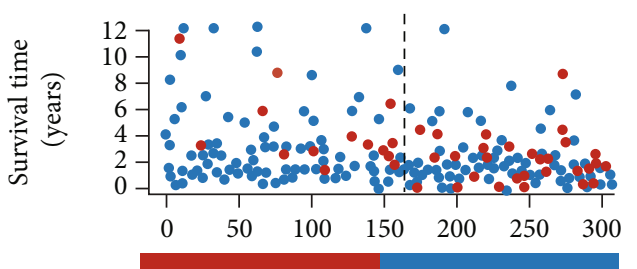

Patients (increasing nomogram score)

- Dead Nomogram risk stratification

- Alive High-risk

Low-risk 


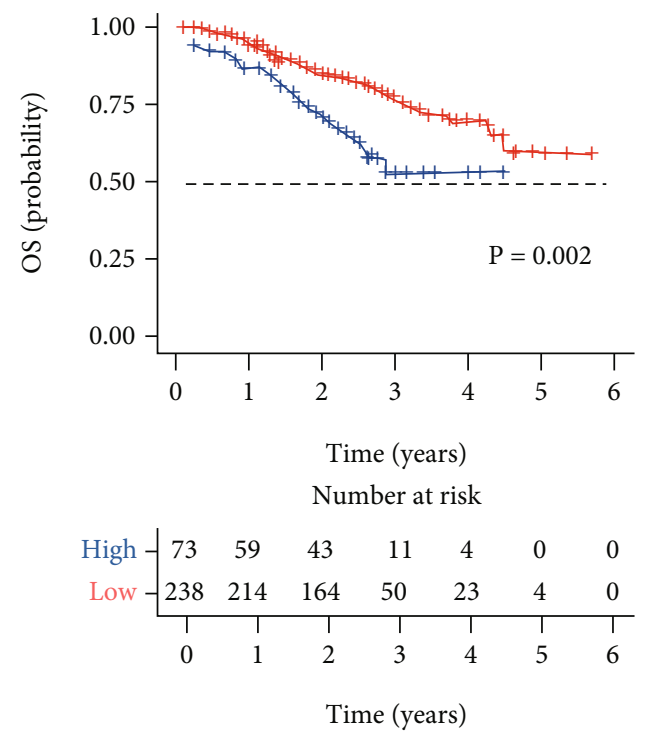

Nomogram risk stratification
+ High
+ Low

(i)

FIgURE 4: Analyses of BACH1 expression on OS at different risks in lung adenocarcinoma cases. (a, d, g) Impact of BACH1 expression on OS in the TCGA dataset, validation cohort 1 (GSE13213), and validation cohort 2 (GSE72094). (b, e, h) Cut-off point selection for risk stratification according to nomogram scores in the TCGA dataset, validation cohort 1 (GSE13213), and validation cohort 2 (GSE72094). (c, f, i) Kaplan-Meier curves of OS at different risks in the training and validation cohort (before PSM). (f, g) Kaplan-Meier curves of CDFS at different risks based on nomogram scores in the TCGA dataset, validation cohort 1 (GSE13213), and validation cohort 2 (GSE72094).

suggested the top ones that connected with its biofunction. As a transcription factor, it apparently participated frequently in protein expression and biosystem of protein expression associated process. The protein targeting enrichment may give us a new view to tumor target therapy. $\mathrm{BACH} 1$ also participates in viral gene transcription and expression, which hints us its connection with some viralinduced tumor, for example, cervical cancer [18].

Lee et al. reported that $\mathrm{BACH} 1$ affected the transcription of electron transport chain (ETC) genes [10], which mainly functioned in the mitochondria. Our big data analysis also yielded similar results, which suggested that BACH1 mainly play vital role in the biological activity in mitochondria. It inhibits the ETC gene transcription and leads to the more available independence from mitochondrial aerobic respiration. What is interested is that $\mathrm{BACH} 1$ may contribute to metastasis via the focal adhesion kinase (FAK), which is an important mediator of cell proliferation, differentiation, and migration [19]. Malignant metastasis normally comes down to the ECM or cellular permeation process [20, 21]; it is not surprising that FAK participates in that, which has also been confirmed by studies from both mouse model and human patients. From the cellular view, BACH1 obviously activates the serine/threonine kinase, which always binds to the transforming growth factor- $\beta$ s (TGF- $\beta$ s). Its activation has been estimated as a vital promoter in neoplasia. Another potential tumor-promoting effect may be its binding to the small GTPase, which contains five subfamily members: Ras, Rho, Rab, Sarl/Arf, and Ran. Among them, Ras plays a vital role in the human neoplasia. They are signaling nodes that are activated in response to a variety of extracellular stimuli. Activated Ras combines with various effectors with different catalytic activities to regulate cytoplasmic signal network, so as to control gene expression, cell proliferation, differentiation, and growth.

KEGG analysis comprehensively recapitulates the bioinformation from both macroscopic and microscopic views, which showed us an excellent data that BACH1 may be an important prognosis factor to the NSCLC. Some NSCLC patients, who burden the mutation of epidermal growth factor receptor (EGFR), have pointers to the target therapy [22] and may also have poor therapeutic effect due to high BACH1 expression.

Besides, the PI3K-Akt signaling pathway, which was known to be a signaling pathway closely related to the occurrence and development of tumors, was also significantly activated in the high BACH1 expression cases. GSEA analysis also showed suppressed oxidative phosphorylation and DNA repair pathway and activated oncogenic pathways such as angiogenesis, IL-6/JAK/STAT3, and TGF- $\beta$ signaling pathways in the high-expression group of BACH1.

ROS is a well-known cancer-related system, which could be generated by neutrophils, macrophages, and even tumor cell itself. Due to this complex and contradictory system in the neoplasia [23], cancer cells engage a relative safe environment to survive and proliferate, which we called TME 


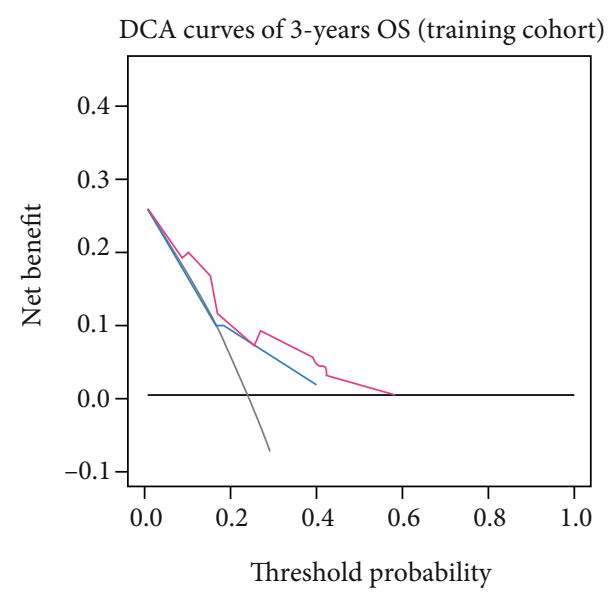

(a)

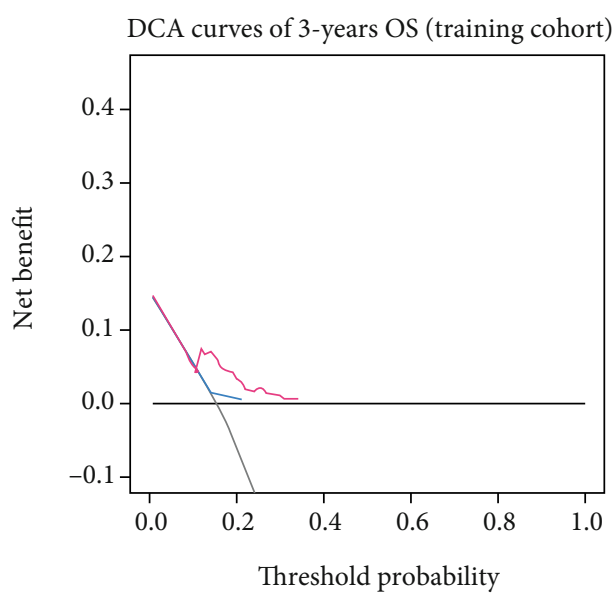

(c)

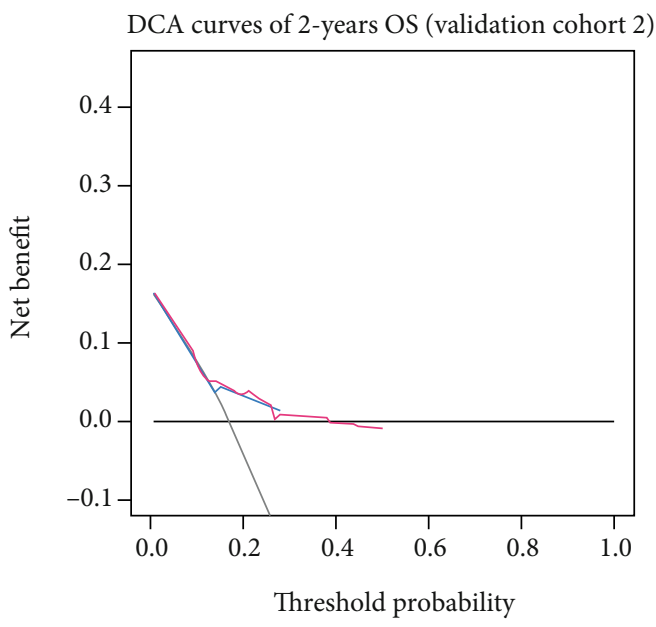

(e)

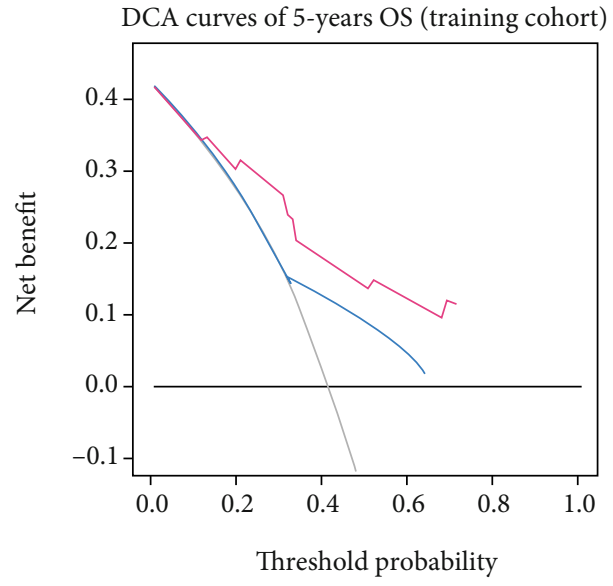

(b)

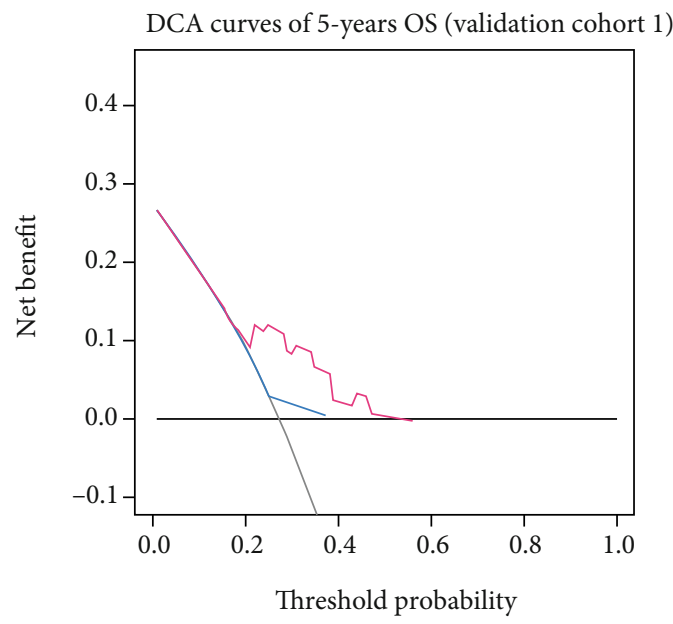

(d)

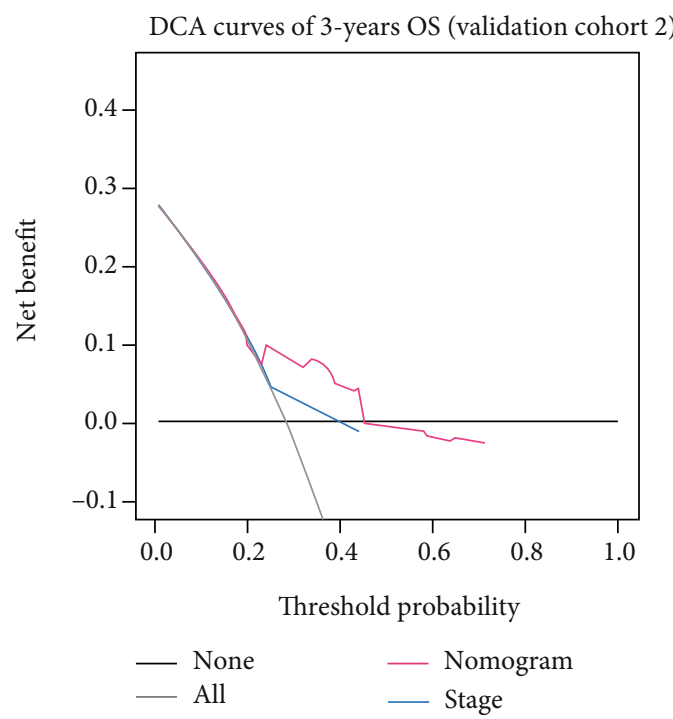

(f)

FIGURE 5: DCA curves of nomogram model compared with pathologic stage. (a, b) DCA curves of 3- and 5-year in the TCGA dataset. (c, d) DCA curves of 3- and 5-year in the validation cohort 1 (GSE13213). (e, f) DCA curves of 2- and 3-year in the validation cohort 2 (GSE72094). 
(tumor microenvironment). Through inhibiting BACH1, ROS prevents the malignant proliferation and metastasis; however, BACH1-activated FAK [24] may help the cells to attach to extracellular matrix, thus contributes to the oxidative environment in the solid tumor, which in turn can help $\mathrm{BACH} 1$ restrain the ETC gene expression. At last, these factors help to construct a neoplasia-fitted hypoxia microenvironment and promote the proceeding of tumor proliferation. BACH1-enriched immune cells can drive immune storm alone or cooperate together. For example, neutrophils promote tumorigenesis via the release of ROS, which contributes to DNA damage [25]. Animal experiment that conducted in zebrafish showed the cooperation of macrophages and neutrophils in neoplasia, in which macrophages can attract neutrophils through ROS-Src family kinase signaling, which hints the important recruitment role of TME to immune cells how macrophages modulate the attachment of immune cells [23].

\section{Conclusions}

In conclusion, our study confirmed the vital role of BACH1 in the prognosis of early-stage lung adenocarcinoma. The orchestration of a complex cell signal network that affects the proliferation and invasion of cancer destines $\mathrm{BACH} 1$ to be a promising predictor of the prognosis of NSCLC and a new potential cancer target.

\section{Data Availability}

The data supporting this study are from previously reported studies and datasets, which have been cited.

\section{Conflicts of Interest}

The authors declare that there is no conflict of interest regarding the publication of this paper.

\section{Authors' Contributions}

Qibin Song and Yi Yao contributed equally and share the corresponding authorship. Qibin Song conceived the study. Jin Zhou was in charge of the manuscript draft. Zheming Liu and Huibo Zhang supervised data collection and analysis. Tianyu Lei, Jiahui Liu, and Yaqi Zhao were responsible for data collection and confirmation. Qibin Song and Yi Yao made substantial revisions to the manuscript.

\section{Acknowledgments}

The study is supported by the National Natural Science Foundation of China (Grant No. 81670123).

\section{References}

[1] R. L. Siegel, K. D. Miller, and A. Jemal, "Cancer statistics, 2019," CA: a Cancer Journal for Clinicians, vol. 69, no. 1, pp. 7-34, 2019.

[2] F. Bray, J. Ferlay, I. Soerjomataram, R. L. Siegel, L. A. Torre, and A. Jemal, "Global cancer statistics 2018: GLOBOCAN esti- mates of incidence and mortality worldwide for 36 cancers in 185 countries," CA: a Cancer Journal for Clinicians, vol. 68, no. 6, pp. 394-424, 2018.

[3] Global Burden of Disease Cancer Collaboration, C. Fitzmaurice, C. Allen et al., "Global, regional, and national cancer incidence, mortality, years of life lost, years lived with disability, and disability-adjusted life-years for 32 cancer groups, 1990 to 2015: a systematic analysis for the Global Burden of Disease Study," JAMA Oncology, vol. 3, no. 4, pp. 524548, 2017.

[4] P. Goldstraw, D. Ball, J. R. Jett et al., "Non-small-cell lung cancer," Lancet, vol. 378, no. 9804, pp. 1727-1740, 2011.

[5] K. Igarashi, T. Kurosaki, and R. Roychoudhuri, "BACH transcription factors in innate and adaptive immunity," Nature Reviews. Immunology, vol. 17, no. 7, pp. 437-450, 2017.

[6] A. W. Reinke, J. Baek, O. Ashenberg, and A. E. Keating, "Networks of bZIP protein-protein interactions diversified over a billion years of evolution," Science, vol. 340, no. 6133, pp. 730-734, 2013.

[7] T. Oyake, K. Itoh, H. Motohashi et al., "Bach proteins belong to a novel family of BTB-basic leucine zipper transcription factors that interact with $\mathrm{MafK}$ and regulate transcription through the NF-E2 site," Molecular and Cellular Biology, vol. 16, no. 11, pp. 6083-6095, 1996.

[8] H. Peinado, H. Zhang, I. R. Matei et al., "Pre-metastatic niches: organ-specific homes for metastases," Nature Reviews. Cancer, vol. 17, no. 5, pp. 302-317, 2017.

[9] X. Z. West, N. L. Malinin, A. A. Merkulova et al., "Oxidative stress induces angiogenesis by activating TLR2 with novel endogenous ligands," Nature, vol. 467, no. 7318, pp. 972976, 2010.

[10] J. Lee, A. E. Yesilkanal, J. P. Wynne et al., "Effective breast cancer combination therapy targeting $\mathrm{BACH} 1$ and mitochondrial metabolism," Nature, vol. 568, no. 7751, pp. 254-258, 2019.

[11] L. Lignitto, S. E. LeBoeuf, H. Homer et al., "Nrf2 activation promotes lung cancer metastasis by inhibiting the degradation of Bach1," Cell, vol. 178, no. 2, pp. 316-329.e18, 2019.

[12] C. Wiel, K. le Gal, M. X. Ibrahim et al., "BACH1 stabilization by antioxidants stimulates lung cancer metastasis," Cell, vol. 178, no. 2, pp. 330-345.e22, 2019.

[13] G. Yu, L.-G. Wang, Y. Han, and Q.-Y. He, “clusterProfiler: an $\mathrm{R}$ package for comparing biological themes among gene clusters," OMICS: a Journal of Integrative Biology, vol. 16, no. 5, pp. 284-287, 2012.

[14] A. J. Vickers, A. M. Cronin, E. B. Elkin, and M. Gonen, "Extensions to decision curve analysis, a novel method for evaluating diagnostic tests, prediction models and molecular markers," BMC Medical Informatics and Decision Making, vol. 8, no. 1, 2008.

[15] B. Xu, K. H. Song, Y. Yao et al., "Individualized model for predicting COVID-19 deterioration in patients with cancer: a multicenter retrospective study," Cancer Science, vol. 112, no. 6, pp. 2522-2532, 2021.

[16] S. G. da Cunha, F. A. Shepherd, and M. S. Tsao, "EGFR mutations and lung cancer," Annual Review of Pathology, vol. 6, no. 1, pp. 49-69, 2011.

[17] S. L. Topalian, J. M. Taube, R. A. Anders, and D. M. Pardoll, "Mechanism-driven biomarkers to guide immune checkpoint blockade in cancer therapy," Nature Reviews. Cancer, vol. 16, no. 5, pp. 275-287, 2016. 
[18] P. A. Cohen, A. Jhingran, A. Oaknin, and L. Denny, "Cervical cancer," Lancet, vol. 393, no. 10167, pp. 169-182, 2019.

[19] S. K. Mitra, D. A. Hanson, and D. D. Schlaepfer, "Focal adhesion kinase: in command and control of cell motility," Nature Reviews. Molecular Cell Biology, vol. 6, no. 1, pp. 56-68, 2005.

[20] B. Psaila and D. Lyden, "The metastatic niche: adapting the foreign soil," Nature Reviews. Cancer, vol. 9, no. 4, pp. 285293, 2009.

[21] R. N. Kaplan, R. D. Riba, S. Zacharoulis et al., "VEGFR1positive haematopoietic bone marrow progenitors initiate the pre- metastatic niche," Nature, vol. 438, no. 7069, pp. 820-827, 2005.

[22] A. Midha, S. Dearden, and R. McCormack, "EGFR mutation incidence in non-small-cell lung cancer of adenocarcinoma histology: a systematic review and global map by ethnicity (mutMapII)," American Journal of Cancer Research, vol. 5, no. 9, pp. 2892-2911, 2015.

[23] S. Tauzin, T. W. Starnes, F. B. Becker, P. Y. Lam, and A. Huttenlocher, "Redox and Src family kinase signaling control leukocyte wound attraction and neutrophil reverse migration," The Journal of Cell Biology, vol. 207, no. 5, pp. 589-598, 2014.

[24] F. J. Sulzmaier, C. Jean, and D. D. Schlaepfer, "FAK in cancer: mechanistic findings and clinical applications," Nature Reviews. Cancer, vol. 14, no. 9, pp. 598-610, 2014.

[25] D. R. Powell and A. Huttenlocher, "Neutrophils in the tumor microenvironment," Trends in Immunology, vol. 37, no. 1, pp. 41-52, 2016. 